Article

\title{
Dietary Niacin and Open-Angle Glaucoma: The Korean National Health and Nutrition Examination Survey
}

\author{
Kyoung In Jung, Yong Chan Kim and Chan Kee Park* \\ Department of Ophthalmology, Seoul St. Mary's Hospital, College of Medicine, The Catholic University of \\ Korea, 222 Banpo-daero, Seocho-ku, Seoul 137-701, Korea; ezilean@hanmail.net (K.I.J.); \\ mychan2265@gmail.com (Y.C.K.) \\ * Correspondence: ckpark@catholic.ac.kr; Tel.: +82-2-2258-6199; Fax: +82-2-599-7405
}

Received: 9 January 2018; Accepted: 17 March 2018; Published: 22 March 2018

\begin{abstract}
Glaucoma is a leading cause of loss of sight. High intraocular pressure (IOP) is the most critical risk factor. However, glaucoma develops even within a normal IOP range. Normal tension glaucoma (NTG) is more common in Asia, whereas high tension glaucoma is more common in Western countries. The pathogenesis of glaucoma, especially NTG, is poorly understood. We evaluated the correlation between dietary nutrient intake and glaucoma using data from subjects $\geq 40$ years old from the ongoing, nationwide, population-based study, the Korean National Health and Nutrition Examination Survey V (2008-2012). Dietary intake was determined using the $24 \mathrm{~h}$ recall method. Fiber (g/day), ash (g/day), calcium (mg/day), phosphorus (mg/day), iron (mg/day), sodium (mg/day), potassium (mg/day), $\beta$-Carotene ( $\mu \mathrm{g} /$ day), retinol ( $\mu \mathrm{g} /$ day), vitamin A ( $\mu \mathrm{g}$ Retinol Equivalents/day), thiamine (mg/day), riboflavin ( $\mathrm{mg} /$ day), niacin (mg/day), and vitamin $\mathrm{C}$ ( $\mathrm{mg} /$ day) were included in nutrient intake data. All nutrient intake was divided into quartiles. The mean IOP did not differ according to quartiles from any nutrients (all $p>0.05$ ). After adjusting for age, gender, income status, education level, smoking, alcohol consumption, physical activity, diabetes, hypertension, IOP, and total energy, the intake of niacin was associated with glaucoma $(p=0.013)$. Among subjects with IOP $\leq 21 \mathrm{mmHg}$, only niacin was related to glaucoma in a multivariate analysis $(p=0.022)$. Dietary nutrient intake was associated with open-angle glaucoma independent of IOP. Individuals with NTG showed lower intake of niacin among nutrients. This finding suggests the possibility that proper diet counseling may be another modifiable factor, aside from IOP, particularly among patients with NTG.
\end{abstract}

Keywords: glaucoma; niacin; normal tension glaucoma; nutrients

\section{Introduction}

Glaucoma is a leading cause of loss of sight, affecting more than 70 million people worldwide [1,2]. The pathogenesis of glaucoma is not fully established, although intraocular pressure (IOP) is the most critical and treatable risk factor. Glaucoma develops even within a normal IOP range and can progress, even with a substantial decrease in IOP. Excitotoxicity, unstable blood flow and oxidative stress can also affect the development and progression of glaucoma [3].

A family history of glaucoma is one of the risk factors for primary open-angle glaucoma (POAG) [4]. Family members have a high probability of having common genetic susceptibility or similar lifestyles, such as diet. Given those findings, different genes or environmental factors may be linked to the development of glaucoma. To date, identified gene mutations are responsible for $<10 \%$ of POAG in the general population [5]. An active interest in environmental or lifestyle factors, such as diet patterns, and their association with glaucoma may be needed to understand the pathogenesis of glaucoma. 
The most common type of open-angle glaucoma in Asian countries is normal tension glaucoma (NTG), whereas high tension glaucoma is more common in the United States and Europe [6]. Staple foods and dietary habits are distinctly different between Asian and Caucasian individuals, even though the prevalence of a Westernized diet has increased in Asian countries. The proportion of NTG in a Japanese American population was $80.4 \%$ of all POAG cases, which was lower than $92.3 \%$ in the Tajimi Study conducted in Japan. One of the reasons for the lower rate of NTG in the Japanese American population may be differences in environmental factors such as nutrition $[7,8]$.

Accumulating clinical evidence suggests a possible association between nutrition or diet composition and POAG [9-13], although a few studies did not find this correlation [14,15]. Lower ingestion of retinol equivalents, vitamins $\mathrm{B} 2$ and $\mathrm{C}$, a higher ratio of $n-3$ to $n-6$ polyunsaturated fats, and a lower ingestion of certain fruits or vegetables have been found to be related to the risk for POAG [9-13]. Among nutrients, vitamins with anti-oxidant activity are of interest, as they may relieve oxidative stress in the pathogenesis of glaucoma [16,17].

To date, no study has addressed the relationship between diverse dietary nutrients and glaucoma in Asian countries. In this study, we investigated the potential impact of dietary nutrients on glaucoma using the Korean National Health and Nutrition Examination Survey (KNHANES), a large population-based study. Determination of modifiable risk factors, aside from IOP, can provide other options for treating glaucoma patients showing progression of the disease, despite a relatively low IOP range.

\section{Methods}

The KNHANES is an ongoing nationwide epidemiological study conducted by the Division of Chronic Disease Control and Prevention, Ministry of Health and Welfare, with the approval of its Institutional Review Board. The survey follows the tenets of the Declaration of Helsinki for biomedical research involving humans. Written informed consent was provided by all participants. The KNHANES uses a stratified, multistage, probability cluster survey with a rolling survey model. All participants are randomly chosen from randomly assigned districts of cities and provinces in South Korea.

Among the 37,982 participants in the KNHANES 2008-2012 IV-V, those who were younger than 40 years old or who did not complete an ophthalmic examination were excluded. Other exclusion criteria were as follows: participants with a narrow angle using the Van Herick method (peripheral anterior chamber depth $\leq 4 / 1$ of peripheral corneal thickness); those whose fundus photograph displayed geographic atrophy or signs of wet age-related macular degeneration, such as retinal pigment epithelial (RPE) detachment, serous detachment of the sensory retina, sub-retinal or sub-RPE hemorrhage, or sub-retinal fibrous scars; those who had not completed the nutritional survey; and those with missing data.

Data on anthropometrics and demographic characteristics, including socioeconomic status and levels of education and physical activity, were collected. Participants were divided into nondrinkers, mild drinkers ( $\leq$ one time per month), moderate drinkers (>one time and $\leq$ four times per month) and heavy drinkers ( $>$ four times per month). Smoking status was classified into nonsmokers, former smokers, and current smokers. Physical activity (PA) scores were categorized based on the International PA Questionnaire guidelines. Moderate physical activity was defined as the performance of moderate-intensity physical activity for $\geq 20 \mathrm{~min} \mathrm{3-4} \mathrm{times/week.} \mathrm{The} \mathrm{definition} \mathrm{of} \mathrm{moderate}$ intensity was exercise that induced a mild elevation in breathing or heart rate for at least $10 \mathrm{~min}$. Data on medical comorbidities, such as diabetes mellitus and hypertension, were also collected as potential confounding variables. Diabetes was defined as a fasting blood glucose level higher than $126 \mathrm{mg} / \mathrm{dL}$ or the current use of a systemic antidiabetic treatment. Systemic hypertension was defined as a systolic blood pressure (BP) $>160 \mathrm{mmHg}$, diastolic $\mathrm{BP}>90 \mathrm{mmHg}$, or the current use of a systemic antihypertensive medication. 


\subsection{Ophthalmologic Measurements}

Participants underwent ophthalmological examinations, including slit-lamp examinations and fundus photography. Ophthalmologists measured IOP using a Goldmann applanation tonometer (Haag-streit, Inc., Bern, Switzerland).

Participants with elevated IOP $(\geq 22 \mathrm{mmHg}$ ) or a glaucomatous optic disc were examined using frequency-doubling technology (Humphrey Matrix; Carl Zeiss Meditec, Inc., Jena, Germany) using the N-30-1 program. A glaucomatous optic disc refers to any of the following: a horizontal or vertical cup-to-disc ratio (vCDR) of $\geq 0.5$, the appearance of optic disc hemorrhage, the presence of a retinal nerve fiber layer defect (RNFL), or a violation of the ISNT rule (neuroretinal rim thickness in the order of inferior $>$ superior $>$ nasal $>$ temporal). Subjects underwent the test again when fixation errors or false-positive errors were $>33 \%$. A glaucoma diagnosis was made using modified ISGEO criteria $[18,19]$ : when both fixation errors and false-positive errors were $33 \%$ or less, category 1 requires a glaucomatous visual field defect with a CDR of $\geq 0.7$, asymmetry of a vCDR of $\geq 0.2$, or the presence of an RNFL defect $[18,19]$. When the visual field was not available or was unsatisfactory, subjects were included in category 2 if their vCDR was $\geq 0.9$, the vCDR asymmetry was $\geq 0.3$ or if they had an RNFL loss with a violation of the ISNT rule [19]. If examinations of the optic disc and visual field tests were not possible, category 3 required a visual acuity of $<3 / 60$ and IOP exceeding $21 \mathrm{mmHg}$ [18]. Subgroup analysis was done within subjects with NTG; NTG was defined as an IOP was $\leq 21 \mathrm{mmHg}$.

\subsection{Assessment of Nutrient Intake}

Dietary intake was determined using the $24 \mathrm{~h}$ recall method by trained staff. All subjects were instructed to continue their ordinary diets before the dietary evaluation. The information gained on holidays or weekends was not excluded. Nutrient intake was calculated on the basis of the nutrient concentrations in foods using the Korean Food Composition Table, which was devised by the Korean National Rural Resources Development Institute [20]. Fiber (g/day), ash (g/day), calcium (mg/day), phosphorus (mg/day), iron ( $\mathrm{mg} /$ day), sodium (mg/day), potassium (mg/day), $\beta$-Carotene $(\mu \mathrm{g} /$ day), retinol ( $\mu \mathrm{g}$ /day), thiamine ( $\mathrm{mg} /$ day), riboflavin $(\mathrm{mg} /$ day), niacin $(\mathrm{mg} /$ day), and Vitamin $\mathrm{C}(\mathrm{mg} /$ day) were included in nutrient intake data. vitamin A ( $\mu$ g Retinol Equivalents (RE)/day) was calculated by summing retinol $(\mu \mathrm{g} /$ day) and $\beta$-Carotene/ 6 ( $\mu \mathrm{g} /$ day). The individuals were divided into quartiles of total intake per day for each nutrient $(\mathrm{Q} 1, \mathrm{Q} 2, \mathrm{Q} 3$ and Q4). If individuals had taken any kind of supplements consecutively more than 2 weeks over the past year or more than one time per week over the past month, they were regarded as taking supplements.

\subsection{Statistical Analyses}

SAS Software (version 9.2; SAS Institute, Inc., Cary, NC, USA) was used for statistical analyses to reflect sampling weights and to offer nationally representative prevalence estimates. Demographic factors between subjects with and without glaucoma were compared using Student's $t$-tests for continuous parameters and $\chi^{2}$ tests for categorical variables. Continuous and categorical parameters are described as the mean \pm standard error and percentage, respectively. Multivariate adjusted logistic analysis was performed to investigate factors associated with glaucoma. We analyzed the subset of data after excluding subjects taking supplements. Factors with a difference of $p<0.1$ between the two groups, IOP, and total energy were entered into a multivariate analysis. Odds ratios (ORs) and 95\% confidence intervals were calculated. A $p$ value of $<0.05$ was considered statistically significant.

\section{Results}

Among 37,982 participants in the KNHANES 2008-2012 IV-V, those younger than 40 years old were excluded $(n=17,563)$. Subjects who did not receive an ophthalmic examination were also excluded $(n=95)$. Participants with a narrow angle using the Van Herick method (peripheral anterior chamber depth $\leq 4 / 1$ of peripheral corneal thickness; $n=350$ ), those whose fundus photograph displayed 
geographic atrophy or signs of wet age-related macular degeneration, such as retinal pigment epithelial (RPE) detachment, serous detachment of the sensory retina, sub-retinal or sub-RPE hemorrhage, or sub-retinal fibrous scars $(n=123)$, those who had not completed the nutritional survey $(n=2030)$, and those with missing data $(n=757)$ were excluded. A total 16,770 participants (6902 men, 9868 women) were included in this study (Figure 1).

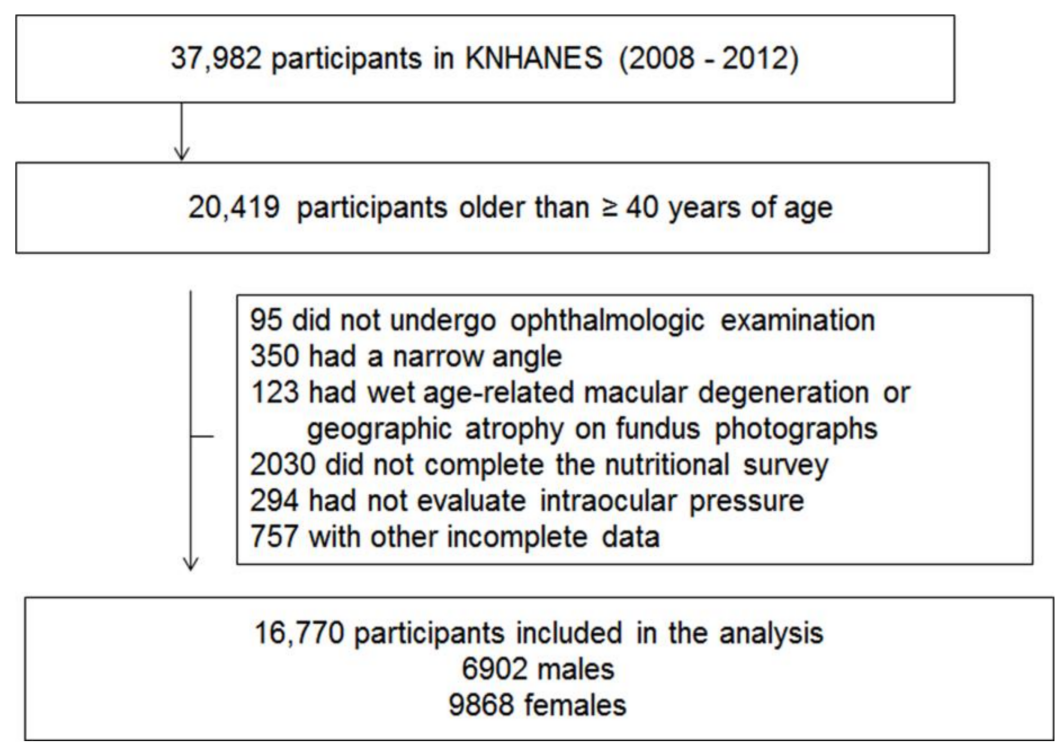

Figure 1. Flow diagram displaying selection of study subjects.

The demographics are shown in Table 1. Seven hundred and seventy-five participants were diagnosed with glaucoma (overall prevalence, $4.11 \%$ ). The proportion of individuals with glaucoma diagnosis made in the absence of visual field defects and/or abnormal optic disc parameters was $47.4 \%$. The average age of participants with glaucoma was $60.7 \pm 0.6$ years old. Subjects with glaucoma were older and less educated, exercised less regularly, were more likely to smoke, have no occupation, and have diabetes or hypertension than those without glaucoma (all $p<0.05$ ). IOP was higher in subjects with glaucoma $(14.7 \pm 0.2 \mathrm{mmHg})$ than in those without glaucoma $(14.0 \pm 0.1 \mathrm{mmHg}, p<0.001)$.

Table 1. Baseline characteristics.

\begin{tabular}{|c|c|c|c|}
\hline \multirow{3}{*}{ Characteristics } & \multicolumn{2}{|c|}{ Glaucoma } & \multirow{3}{*}{$p$ Value } \\
\hline & No & Yes & \\
\hline & $n=15995$ & $n=775$ & \\
\hline Age (years) & $55.2 \pm 0.2$ & $60.7 \pm 0.6$ & $<0.001$ \\
\hline \multicolumn{4}{|l|}{ Gender } \\
\hline Male & $6552(47.83)$ & $350(54.36)$ & 0.006 \\
\hline Female & 9443 (52.17) & $425(45.64)$ & \\
\hline \multicolumn{4}{|l|}{ BMI $\left(\mathrm{kg} / \mathrm{m}^{2}\right)$} \\
\hline Underweight $(<18.5)$ & $492(2.78)$ & $35(4.18)$ & 0.108 \\
\hline Normal weight $(\geq 18.5,<23)$ & $5825(35.69)$ & $296(37.78)$ & \\
\hline Overweight $(\geq 23,<25)$ & $4137(25.86)$ & $198(26.64)$ & \\
\hline Obese $(\geq 25)$ & $5541(35.67)$ & $246(31.40)$ & \\
\hline \multicolumn{4}{|l|}{ Income status } \\
\hline Lowest & 4155 (20.39) & $296(29.25)$ & $<0.001$ \\
\hline Lower middle & $3985(25.60)$ & $183(24.31)$ & \\
\hline Higher middle & $3794(26.20)$ & $136(21.69)$ & \\
\hline Highest & $4061(27.80)$ & $160(24.75)$ & \\
\hline
\end{tabular}


Table 1. Cont

\begin{tabular}{|c|c|c|c|}
\hline \multirow{3}{*}{ Characteristics } & \multicolumn{2}{|c|}{ Glaucoma } & \multirow{3}{*}{$p$ Value } \\
\hline & \multirow{2}{*}{$\begin{array}{c}\text { No } \\
n=15995\end{array}$} & \multirow{2}{*}{$\begin{array}{c}\text { Yes } \\
n=775\end{array}$} & \\
\hline & & & \\
\hline Education & & & \\
\hline Elementary school or less & $6199(30.74)$ & 409 (44.64) & $<0.001$ \\
\hline Middle school graduate & $2466(15.65)$ & $98(12.31)$ & \\
\hline High school graduate & $4503(32.79)$ & $161(24.42)$ & \\
\hline University graduate or higher & $2827(20.81)$ & $107(18.63)$ & \\
\hline \multicolumn{4}{|l|}{ Occupation } \\
\hline White collar & 2047 (16.55) & $53(9.71)$ & $<0.001$ \\
\hline Blue collar & $7142(47.74)$ & $323(44.42)$ & \\
\hline Inoccupation & $6806(35.71)$ & $399(45.87)$ & \\
\hline \multicolumn{4}{|l|}{ Smoking } \\
\hline Never & $9760(54.90)$ & $437(48.41)$ & 0.015 \\
\hline Former & $3961(29.25)$ & $192(31.19)$ & \\
\hline Current & $2274(15.85)$ & $146(20.41)$ & \\
\hline \multicolumn{4}{|l|}{ Alcohol intake (n) } \\
\hline Non-drink & 5705 (30.22) & $327(35.28)$ & 0.064 \\
\hline$\leq 1$ in month & $4322(26.72)$ & $194(25.44)$ & \\
\hline$\leq 4$ in month & $2697(18.75)$ & $102(14.85)$ & \\
\hline$\geq 2$ in week & $3271(24.32)$ & $152(24.44)$ & \\
\hline \multicolumn{4}{|l|}{ Region of residence } \\
\hline Rural & $6134(33.24)$ & $329(35.37)$ & 0.356 \\
\hline Urban & $9861(66.76)$ & $446(64.63)$ & \\
\hline \multicolumn{4}{|l|}{ Physical activity ( $\geq$ moderate intensity) } \\
\hline No & $12,473(77.72)$ & $632(81.48)$ & 0.047 \\
\hline Yes & $3522(22.28)$ & $143(18.52)$ & \\
\hline \multicolumn{4}{|l|}{ Diabetes } \\
\hline No & $13,798(87.43)$ & $612(79.88)$ & $<0.001$ \\
\hline Yes & $2197(12.57)$ & $163(20.12)$ & \\
\hline \multicolumn{4}{|l|}{ Hypertension } \\
\hline No & $9638(64.21)$ & $370(49.72)$ & $<0.001$ \\
\hline Yes & $6357(35.79)$ & $405(50.28)$ & \\
\hline Intraocular pressure (mmHg) & $14.0 \pm 0.1$ & $14.7 \pm 0.2$ & $<0.001$ \\
\hline
\end{tabular}

Data are presented as mean $\pm \mathrm{SE}, n$ (weighted \%). Statistics were carried out using $t$-test, Rao-Scott Chi-square.

Quartile categories of each nutrient intake are displayed in Table 2.

Table 2. Quartile categories of nutrient intake.

\begin{tabular}{ccccc}
\hline Nutrient & Q1 & Q2 & Q3 & Q4 \\
\hline Total energy intake (kcal) & $<1387.6$ & $\geq 1387.6$ & $\geq 1814.5$ & $\geq 2352.1$ \\
Crude fiber (g/day) & $<4.54$ & $\geq 4.54$ & $\geq 6.77$ & $\geq 9.72$ \\
Ash (g/day) & $<12.53$ & $\geq 12.53$ & $\geq 18.13$ & $\geq 25.30$ \\
Calcium (mg/day) & $<283.08$ & $\geq 283.08$ & $\geq 436.92$ & $\geq 645.29$ \\
Phosphorus (mg/day) & $<807.97$ & $\geq 807.97$ & $\geq 1083.58$ & $\geq 1444.31$ \\
Iron (mg/day) & $<8.63$ & $\geq 8.63$ & $\geq 12.90$ & $\geq 19.00$ \\
Sodium(mg/day) & $<2731.53$ & $\geq 2731.53$ & $\geq 4239.08$ & $\geq 6368.29$ \\
Potassium (mg/day) & $<2005.32$ & $\geq 2005.32$ & $\geq 2824.62$ & $\geq 3884.43$ \\
A-Carotene ( $\mu$ g/day) & $<316.68$ & $\geq 316.68$ & $\geq 602.05$ & $\geq 1050.90$ \\
Retinol ( $\mu$ g/day) & $<1556.89$ & $\geq 1556.89$ & $\geq 3103.72$ & $\geq 5586.47$ \\
Thiamin (mg/day) & $<8.45$ & $\geq 8.45$ & $\geq 41.02$ & $\geq 105.74$ \\
Riboflavin (mg/day) & $<0.78$ & $\geq 0.78$ & $\geq 1.11$ & $\geq 1.57$ \\
Niacin (mg/day) & $<0.67$ & $\geq 0.67$ & $\geq 1.03$ & $\geq 1.49$ \\
Vitamin C (mg/day) & $<10.15$ & $\geq 10.15$ & $\geq 14.42$ & $\geq 20.49$ \\
Vitamin & $<50.48$ & $\geq 50.48$ & $\geq 85.71$ & $\geq 141.21$ \\
\hline
\end{tabular}


With regard to nutrients, intake of crude fiber, ash, calcium, phosphorus, iron, sodium, potassium, vitamin $\mathrm{A}, \beta$-Carotene, retinol, thiamin, riboflavin, niacin, and vitamin $\mathrm{C}$ was associated with glaucoma (all $p<0.05$; Table 3).

Table 3. The association between dietary nutrient intakes and glaucoma.

\begin{tabular}{|c|c|c|c|c|c|c|c|}
\hline \multirow{4}{*}{ Nutrient } & \multirow{2}{*}{\multicolumn{2}{|c|}{$\begin{array}{c}\text { Gender = Male } \\
\text { Glaucoma }\end{array}$}} & \multirow{4}{*}{$p$ Value } & \multirow{4}{*}{ Nutrient } & \multirow{2}{*}{\multicolumn{2}{|c|}{$\begin{array}{c}\text { Gender }=\text { Female } \\
\text { Glaucoma }\end{array}$}} & \multirow{4}{*}{$p$ Value } \\
\hline & & & & & & & \\
\hline & No & Yes & & & No & Yes & \\
\hline & $n=6552$ & $n=350$ & & & $n=9443$ & $n=425$ & \\
\hline Energy intake & & & & Energy intake & & & \\
\hline Q1 & 815 (10.54) & $70(16.87)$ & 0.002 & Q1 & $3600(38.05)$ & $178(40.00)$ & 0.130 \\
\hline Q2 & 1469 (19.86) & $89(25.66)$ & & Q2 & $2814(29.36)$ & $151(33.68)$ & \\
\hline Q3 & 1907 (28.95) & $92(23.43)$ & & Q3 & $2033(21.69)$ & $69(18.65)$ & \\
\hline $\mathrm{Q} 4$ & $2361(40.65)$ & $99(34.04)$ & & $\mathrm{Q} 4$ & $996(10.90)$ & $27(7.66)$ & \\
\hline Crude fiber & & & & Crude fiber & & & \\
\hline Q1 & $1208(17.25)$ & $70(21.55)$ & 0.368 & Q1 & 3014 (31.70) & $165(39.68)$ & 0.009 \\
\hline Q2 & $1597(23.80)$ & $83(24.28)$ & & $\widehat{\mathrm{Q}} 2$ & $2420(26.06)$ & $113(27.20)$ & \\
\hline Q3 & $1807(28.43)$ & $90(26.67)$ & & Q3 & $2070(22.02)$ & $81(18.74)$ & \\
\hline $\mathrm{Q} 4$ & $1940(30.53)$ & $107(27.50)$ & & $\mathrm{Q} 4$ & $1939(20.22)$ & 66 (14.38) & \\
\hline Ash & & & & Ash & & & \\
\hline Q1 & 1107 (14.07) & $79(22.45)$ & 0.003 & Q1 & $3362(34.44)$ & $190(43.36)$ & 0.011 \\
\hline Q2 & $1512(21.62)$ & $77(19.40)$ & & Q2 & $2641(28.29)$ & $114(26.32)$ & \\
\hline Q3 & $1795(28.28)$ & $97(29.71)$ & & Q3 & $2047(21.96)$ & $74(20.27)$ & \\
\hline $\mathrm{Q} 4$ & $2138(36.03)$ & $97(28.44)$ & & $\mathrm{Q} 4$ & 1393 (15.30) & 47 (10.05) & \\
\hline Calcium & & & & Calcium & & & \\
\hline Q1 & 1258 (15.97) & $82(25.02)$ & 0.004 & Q1 & $3231(32.70)$ & $175(40.03)$ & 0.022 \\
\hline Q2 & $1586(24.11)$ & $88(20.81)$ & & $\widehat{\mathrm{Q}} 2$ & $2434(25.90)$ & $106(27.90)$ & \\
\hline Q3 & $1774(28.41)$ & $77(21.55)$ & & Q3 & $2047(22.32)$ & $76(17.09)$ & \\
\hline $\mathrm{Q} 4$ & $1934(31.51)$ & $103(32.62)$ & & $\mathrm{Q} 4$ & 1731 (19.07) & 68 (14.98) & \\
\hline Phosphorus & & & & Phosphorus & & & \\
\hline Q1 & 985 (12.69) & $70(19.80)$ & 0.019 & Q1 & $3480(35.78)$ & $194(44.55)$ & 0.006 \\
\hline Q2 & 1467 (20.87) & $89(22.29)$ & & Q2 & $2708(28.80)$ & $118(27.51)$ & \\
\hline Q3 & $1865(28.56)$ & $91(25.07)$ & & Q3 & 2009 (21.93) & $78(19.82)$ & \\
\hline Q4 & 2235 (37.88) & $100(32.83)$ & & $\mathrm{Q} 4$ & $1246(13.48)$ & $35(8.12)$ & \\
\hline Iron & & & & Iron & & & \\
\hline Q1 & 1235 (16.00) & $84(25.42)$ & 0.004 & Q1 & $3216(32.57)$ & $179(42.70)$ & 0.004 \\
\hline Q2 & $1555(23.38)$ & $80(19.26)$ & & Q2 & $2456(26.67)$ & $108(26.11)$ & \\
\hline$\hat{\mathrm{Q} 3}$ & $1783(28.56)$ & $90(27.55)$ & & $\widehat{\mathrm{Q}} 3$ & $1997(21.91)$ & $75(17.85)$ & \\
\hline Q4 & 1979 (32.06) & $96(27.76)$ & & Q4 & $1774(18.85)$ & $63(13.33)$ & \\
\hline Sodium & & & & Sodium & & & \\
\hline Q1 & 1085 (13.85) & 73 (18.58) & 0.019 & Q1 & $3418(35.00)$ & $171(38.55)$ & 0.584 \\
\hline Q2 & 1499 (21.47) & $69(19.72)$ & & Q2 & $2669(28.31)$ & $120(28.51)$ & \\
\hline Q3 & $1833(28.65)$ & $112(33.91)$ & & Q3 & $1974(21.51)$ & 78 (18.91) & \\
\hline $\mathrm{Q} 4$ & $2135(36.03)$ & $96(27.78)$ & & $\mathrm{Q} 4$ & $1382(15.18)$ & $56(14.03)$ & \\
\hline Potassium & & & & Potassium & & & \\
\hline Q1 & 1154 (15.07) & $76(22.76)$ & 0.014 & Q1 & $3266(33.48)$ & $188(44.12)$ & $<0.001$ \\
\hline Q2 & $1564(22.70)$ & $83(22.04)$ & & Q2 & $2487(27.10)$ & $123(28.56)$ & \\
\hline Q3 & 1827 (28.79) & 99 (29.10) & & Q3 & $2027(21.66)$ & $65(15.86)$ & \\
\hline Q4 & 2007 (33.43) & $92(26.10)$ & & Q4 & 1663 (17.76) & 49 (11.46) & \\
\hline Vitamin A & & & & Vitamin A & & & \\
\hline Q1 & 1466 (19.27) & $94(25.08)$ & 0.150 & Q1 & $2922(29.68)$ & $171(40.06)$ & $<0.001$ \\
\hline Q2 & $1570(24.15)$ & $82(25.32)$ & & Q2 & $2439(25.72)$ & $108(25.86)$ & \\
\hline Q3 & 1745 (27.53) & $84(24.42)$ & & Q3 & $2094(22.85)$ & $92(21.16)$ & \\
\hline $\mathrm{Q} 4$ & 1771 (29.05) & $90(25.18)$ & & $\mathrm{Q} 4$ & $1988(21.74)$ & $54(12.92)$ & \\
\hline$\beta$-Carotene & & & & $\beta$-Carotene & & & \\
\hline Q1 & 1472 (19.69) & 91 (23.99) & 0.247 & Q1 & $2876(29.32)$ & $173(40.70)$ & $<0.001$ \\
\hline Q2 & $1577(24.29)$ & $83(26.92)$ & & Q2 & $2387(25.61)$ & $101(23.44)$ & \\
\hline Q3 & $1724(27.12)$ & 85 (23.53) & & Q3 & $2176(23.29)$ & $89(20.65)$ & \\
\hline $\mathrm{Q} 4$ & 1779 (28.89) & $91(25.56)$ & & $\mathrm{Q} 4$ & $2004(21.78)$ & $62(15.22)$ & \\
\hline Retinol & & & & Retinol & & & \\
\hline Q1 & 1551 (19.54) & $104(24.27)$ & 0.035 & Q1 & 3024 (29.58) & $173(37.05)$ & 0.004 \\
\hline Q2 & $1728(26.03)$ & $101(30.78)$ & & Q2 & $2266(23.68)$ & $108(27.10)$ & \\
\hline Q3 & $1564(24.81)$ & $85(22.90)$ & & Q3 & $2292(25.37)$ & $87(22.49)$ & \\
\hline $\mathrm{Q} 4$ & 1709 (29.62) & $60(22.05)$ & & Q4 & $1861(21.36)$ & 57 (13.36) & \\
\hline
\end{tabular}


Table 3. cont.

\begin{tabular}{|c|c|c|c|c|c|c|c|}
\hline \multirow{4}{*}{ Nutrient } & \multirow{2}{*}{\multicolumn{2}{|c|}{$\begin{array}{c}\text { Gender = Male } \\
\text { Glaucoma }\end{array}$}} & \multirow{4}{*}{$p$ Value } & \multirow{4}{*}{ Nutrient } & \multirow{2}{*}{\multicolumn{2}{|c|}{$\begin{array}{c}\text { Gender }=\text { Female } \\
\text { Glaucoma }\end{array}$}} & \multirow{4}{*}{$p$ Value } \\
\hline & & & & & & & \\
\hline & No & Yes & & & No & Yes & \\
\hline & $n=6552$ & $n=350$ & & & $n=9443$ & $n=425$ & \\
\hline Thiamin & & & \multirow{5}{*}{0.074} & Thiamin & & & \multirow{5}{*}{0.002} \\
\hline Q1 & 1117 (14.11) & 77 (19.68) & & Q1 & 3368 (34.49) & $190(44.33)$ & \\
\hline Q2 & $1535(22.04)$ & 95 (25.03) & & Q2 & $2569(27.60)$ & 125 (28.17) & \\
\hline Q3 & 1779 (27.85) & $88(24.40)$ & & Q3 & 2109 (22.69) & $67(17.41)$ & \\
\hline $\mathrm{Q} 4$ & $2121(36.00)$ & $90(30.89)$ & & $\mathrm{Q} 4$ & $1397(15.22)$ & $43(10.09)$ & \\
\hline Riboflavin & & & \multirow{5}{*}{0.005} & Riboflavin & & & \multirow{5}{*}{$<0.001$} \\
\hline Q1 & 1275 (15.92) & 89 (24.31) & & Q1 & 3260 (32.59) & $200(45.37)$ & \\
\hline Q2 & 1545 (21.93) & $93(24.04)$ & & Q2 & 2646 (27.78) & 117 (27.17) & \\
\hline Q3 & 1709 (27.34) & $86(24.57)$ & & Q3 & $2056(23.26)$ & $60(14.76)$ & \\
\hline $\mathrm{Q} 4$ & $2023(34.81)$ & $82(27.08)$ & & $\mathrm{Q} 4$ & $1481(16.38)$ & $48(12.70)$ & \\
\hline Niacin & & & \multirow{5}{*}{0.007} & Niacin & & & \multirow{5}{*}{$<0.001$} \\
\hline Q1 & 1037 (13.17) & 75 (20.94) & & Q1 & 3471 (35.13) & $217(48.88)$ & \\
\hline Q2 & 1518 (21.49) & $89(23.47)$ & & Q2 & $2657(28.18)$ & $115(27.81)$ & \\
\hline Q3 & $1798(27.42)$ & $101(25.91)$ & & Q3 & $2083(23.08)$ & $61(15.88)$ & \\
\hline Q4 & 2199 (37.92) & 85 (29.69) & & $\mathrm{Q} 4$ & 1232 (13.61) & $32(7.44)$ & \\
\hline Vitamin C & & & \multirow{5}{*}{0.360} & Vitamin C & & & \multirow{5}{*}{0.008} \\
\hline Q1 & 1445 (20.03) & $83(22.78)$ & & Q1 & $2812(29.17)$ & $159(37.95)$ & \\
\hline Q2 & $1662(24.72)$ & $90(27.81)$ & & Q2 & $2352(25.10)$ & $114(25.58)$ & \\
\hline Q3 & $1748(27.91)$ & $94(26.41)$ & & Q3 & $2100(22.50)$ & $84(19.02)$ & \\
\hline Q4 & $1697(27.35)$ & $83(23.00)$ & & Q4 & $2179(23.23)$ & 68 (17.46) & \\
\hline
\end{tabular}

Data are presented as $n$ (weighted \%). Statistics were carried out using $t$-test, Rao-Scott Chi-square test.

Multivariate analyses (model 1) adjusted for age, gender, income status, education level, smoking, alcohol consumption, physical activity, diabetes, hypertension, IOP, and total energy, showed intake of niacin was correlated with glaucoma ( $p=0.013$; Table 4$)$. In a partially adjusted model (all covariates in model 1 but IOP), overall results were similar to those in model 1 multivariate analyses. 
Table 4. Odds ratios of nutritional factors for glaucoma.

\begin{tabular}{|c|c|c|c|c|c|c|c|c|c|}
\hline \multirow{2}{*}{ Nutrient } & \multicolumn{2}{|c|}{ Unadjusted } & \multicolumn{2}{|c|}{ Model 1} & \multirow{2}{*}{ Nutrient } & \multicolumn{2}{|c|}{ Unadjusted } & \multicolumn{2}{|c|}{ Model 1} \\
\hline & OR (95\% CI) & $p$ for Trend & OR (95\% CI) & $p$ for Trend & & OR (95\% CI) & $p$ for Trend & OR (95\% CI) & $p$ for Trend \\
\hline Crude fiber & & \multirow{5}{*}{0.005} & & \multirow{5}{*}{0.221} & Vitamin A & & \multirow{5}{*}{$<0.001$} & & \multirow{5}{*}{0.130} \\
\hline Q1 & Reference & & Reference & & Q1 & Reference & & Reference & \\
\hline Q2 & $0.85(0.67-1.08)$ & & $0.96(0.74-1.23)$ & & Q2 & $0.79(0.63-1.01)$ & & $0.99(0.78-1.26)$ & \\
\hline Q3 & $0.76(0.59-0.98)$ & & $0.90(0.69-1.17)$ & & Q3 & $0.71(0.55-0.91)$ & & $0.93(0.70-1.22)$ & \\
\hline Q4 & $0.71(0.55-0.91)$ & & $0.85(0.63-1.13)$ & & Q4 & $0.60(0.46-0.78)$ & & $0.81(0.61-1.07)$ & \\
\hline Ash & & \multirow{5}{*}{0.001} & & \multirow{5}{*}{0.369} & $\beta$-Carotene & & \multirow{5}{*}{$<0.001$} & & \multirow{5}{*}{0.220} \\
\hline Q1 & Reference & & Reference & & Q1 & Reference & & Reference & \\
\hline Q2 & $0.69(0.54-0.89)$ & & $0.82(0.62-1.07)$ & & Q2 & $0.79(0.62-1.02)$ & & $0.97(0.76-1.24)$ & \\
\hline Q3 & $0.79(0.61-1.01)$ & & $0.95(0.72-1.27)$ & & Q3 & $0.69(0.53-0.90)$ & & $0.89(0.68-1.17)$ & \\
\hline$\widehat{Q} 4$ & $0.61(0.47-0.80)$ & & $0.79(0.55-1.14)$ & & $\widehat{Q} 4$ & $0.65(0.50-0.83)$ & & $0.85(0.65-1.12)$ & \\
\hline Calcium & & \multirow{5}{*}{0.020} & & \multirow{5}{*}{0.615} & Retinol & & \multirow{5}{*}{$<0.001$} & & \multirow{5}{*}{0.192} \\
\hline Q1 & Reference & & Reference & & Q1 & & & Reference & \\
\hline$\widehat{\mathrm{Q}} 2$ & $0.74(0.58-0.95)$ & & $0.89(0.68-1.16)$ & & Q2 & $0.97(0.76-1.22)$ & & $1.14(0.90-1.44)$ & \\
\hline Q3 & $0.60(0.46-0.78)$ & & $0.75(0.56-0.99)$ & & Q3 & $0.75(0.59-0.95)$ & & $0.99(0.76-1.27)$ & \\
\hline Q4 & $0.76(0.58-1.00)$ & & $0.98(0.72-1.33)$ & & $\mathrm{Q} 4$ & $0.59(0.45-0.78)$ & & $0.84(0.63-1.13)$ & \\
\hline Phosphorus & & \multirow{5}{*}{0.006} & & \multirow{5}{*}{0.439} & Thiamin & & \multirow{5}{*}{0.002} & & \multirow{5}{*}{0.366} \\
\hline Q1 & Reference & & Reference & & Q1 & Reference & & Reference & \\
\hline Q2 & $0.79(0.62-1.00)$ & & $0.87(0.66-1.14)$ & & Q2 & $0.85(0.66-1.09)$ & & $0.97(0.74-1.28)$ & \\
\hline Q3 & $0.72(0.56-0.93)$ & & $0.85(0.62-1.17)$ & & $\widehat{\mathrm{Q}} 3$ & $0.67(0.52-0.88)$ & & $0.84(0.61-1.15)$ & \\
\hline$\widehat{Q} 4$ & $0.68(0.51-0.91)$ & & $0.86(0.55-1.33)$ & & $\widehat{Q} 4$ & $0.68(0.51-0.90)$ & & $0.88(0.60-1.30)$ & \\
\hline Iron & & \multirow{5}{*}{0.001} & & \multirow{5}{*}{0.075} & Riboflavin & & \multirow{5}{*}{$<0.001$} & & \multirow{5}{*}{0.104} \\
\hline Q1 & Reference & & Reference & & Q1 & Reference & & Reference & \\
\hline Q2 & $0.66(0.52-0.84)$ & & $0.77(0.59-0.99)$ & & $\widehat{\mathrm{Q}} 2$ & $0.74(0.58-0.94)$ & & $0.88(0.68-1.14)$ & \\
\hline$\widehat{\mathrm{Q}} 3$ & $0.68(0.52-0.90)$ & & $0.81(0.60-1.10)$ & & Q3 & $0.58(0.45-0.75)$ & & $0.74(0.55-1.00)$ & \\
\hline Q4 & $0.62(0.48-0.82)$ & & $0.72(0.52-1.00)$ & & Q4 & $0.59(0.45-0.78)$ & & $0.79(0.54-1.15)$ & \\
\hline Sodium & & \multirow{5}{*}{0.110} & & \multirow{5}{*}{0.452} & Niacin & & & & \\
\hline Q1 & Reference & & Reference & & Q1 & Reference & & Reference & \\
\hline Q2 & $0.85(0.67-1.09)$ & & $1.00(0.77-1.30)$ & & Q2 & $0.75(0.58-0.95)$ & $<0.001$ & $0.80(0.61-1.06)$ & 0.013 \\
\hline Q3 & $0.98(0.77-1.24)$ & & $1.22(0.94-1.58)$ & & Q3 & $0.62(0.48-0.80)$ & & $0.69(0.50-0.95)$ & \\
\hline Q4 & $0.77(0.59-0.99)$ & & $1.04(0.76-1.43)$ & & Q4 & $0.57(0.42-0.76)$ & & $0.60(0.40-0.92)$ & \\
\hline
\end{tabular}


Table 4. Cont.

\begin{tabular}{|c|c|c|c|c|c|c|c|c|c|}
\hline \multirow{2}{*}{ Nutrient } & \multicolumn{2}{|c|}{ Unadjusted } & \multicolumn{2}{|c|}{ Model 1} & \multirow{2}{*}{ Nutrient } & \multicolumn{2}{|c|}{ Unadjusted } & \multicolumn{2}{|c|}{ Model 1} \\
\hline & OR $(95 \%$ CI $)$ & $p$ for Trend & OR $(95 \% \mathrm{CI})$ & $p$ for Trend & & OR $(95 \% \mathrm{CI})$ & $p$ for Trend & OR $(95 \% \mathrm{CI})$ & $p$ for Trend \\
\hline Potassium & & & & & Vitamin C & & & & \\
\hline Q1 & Reference & & Reference & & Q1 & Reference & & Reference & \\
\hline Q2 & $0.76(0.59-0.97)$ & $<0.001$ & $0.88(0.67-1.16)$ & 0.090 & Q2 & $0.90(0.70-1.15)$ & 0.004 & $1.08(0.84-1.39)$ & 0.422 \\
\hline Q3 & $0.70(0.53-0.92)$ & & $0.83(0.61-1.14)$ & & Q3 & $0.77(0.59-1.00)$ & & $0.98(0.74-1.28)$ & \\
\hline$\widehat{\mathrm{Q}} 4$ & $0.58(0.44-0.77)$ & & $0.72(0.49-1.05)$ & & $\widehat{\mathrm{Q}} 4$ & $0.68(0.52-0.89)$ & & $0.90(0.67-1.22)$ & \\
\hline
\end{tabular}

Data are presented OR $(95 \% \mathrm{CI})$. Statistics were carried out using Logistic regression. Model 1 was adjusted for age, gender, income, education, occupation, smoking, drink, physical activity, diabetes, hypertension, intraocular pressure, total energy.CI, confidence interval; OR, odds ratio. 
IOP distributions by nutrient quartiles are displayed in Table 5. The mean IOP did not differ according to nutrient quartiles.

Table 5. Intraocular pressure according to quartiles of nutrient intake.

\begin{tabular}{|c|c|c|c|}
\hline Nutrient & Intraocular Pressure (mmHg) & Nutrient & Intraocular Pressure $(\mathrm{mmHg})$ \\
\hline Crude fiber & & Vitamin A & \\
\hline Q1 & $13.95 \pm 0.07$ & Q1 & $14.05 \pm 0.07$ \\
\hline Q2 & $14.00 \pm 0.06$ & Q2 & $13.94 \pm 0.07$ \\
\hline Q3 & $14.08 \pm 0.07$ & Q3 & $14.01 \pm 0.07$ \\
\hline Q4 & $13.97 \pm 0.07$ & Q4 & $14.00 \pm 0.07$ \\
\hline$p$ for trend & 0.532 & $p$ for trend & 0.701 \\
\hline Ash & & $\beta$-Carotene & \\
\hline Q1 & $13.95 \pm 0.06$ & Q1 & $14.05 \pm 0.07$ \\
\hline $\mathrm{Q} 2$ & $13.97 \pm 0.07$ & $\mathrm{Q} 2$ & $13.99 \pm 0.07$ \\
\hline Q3 & $14.10 \pm 0.07$ & Q3 & $14.00 \pm 0.06$ \\
\hline $\mathrm{Q} 4$ & $13.98 \pm 0.06$ & $\mathrm{Q} 4$ & $13.97 \pm 0.07$ \\
\hline$p$ for trend & 0.339 & $p$ for trend & 0.400 \\
\hline $\mathrm{Ca}$ & & Retinol & \\
\hline Q1 & $13.98 \pm 0.07$ & Q1 & $13.90 \pm 0.06$ \\
\hline Q2 & $13.99 \pm 0.06$ & Q2 & $14.03 \pm 0.07$ \\
\hline Q3 & $14.07 \pm 0.07$ & Q3 & $14.07 \pm 0.06$ \\
\hline $\mathrm{Q} 4$ & $13.96 \pm 0.07$ & $\hat{\mathrm{Q}} 4$ & $14.01 \pm 0.07$ \\
\hline$p$ for trend & 0.914 & $p$ for trend & 0.135 \\
\hline Phosphorus & & Thiamin & \\
\hline Q1 & $13.95 \pm 0.07$ & Q1 & $13.92 \pm 0.07$ \\
\hline Q2 & $13.97 \pm 0.06$ & Q2 & $14.01 \pm 0.07$ \\
\hline$\widehat{\mathrm{Q} 3}$ & $14.06 \pm 0.06$ & Q3 & $13.99 \pm 0.07$ \\
\hline Q4 & $14.03 \pm 0.07$ & Q4 & $14.09 \pm 0.07$ \\
\hline$p$ for trend & 0.186 & $p$ for trend & 0.054 \\
\hline Iron & & Riboflavin & \\
\hline Q1 & $13.96 \pm 0.07$ & Q1 & $13.97 \pm 0.06$ \\
\hline Q2 & $14.02 \pm 0.07$ & Q2 & $13.93 \pm 0.07$ \\
\hline Q3 & $14.02 \pm 0.06$ & Q3 & $14.04 \pm 0.07$ \\
\hline $\mathrm{Q} 4$ & $14.01 \pm 0.07$ & $\mathrm{Q} 4$ & $14.07 \pm 0.07$ \\
\hline$p$ for trend & 0.551 & $p$ for trend & 0.089 \\
\hline Sodium & & Niacin & \\
\hline Q1 & $13.97 \pm 0.06$ & Q1 & $13.98 \pm 0.07$ \\
\hline Q2 & $13.95 \pm 0.07$ & Q2 & $13.95 \pm 0.06$ \\
\hline Q3 & $14.09 \pm 0.07$ & Q3 & $14.00 \pm 0.06$ \\
\hline $\mathrm{Q} 4$ & $14.00 \pm 0.07$ & $\mathrm{Q} 4$ & $14.08 \pm 0.07$ \\
\hline$p$ for trend & 0.331 & $p$ for trend & 0.195 \\
\hline Potassium & & Vitamin C & \\
\hline Q1 & $13.96 \pm 0.07$ & Q1 & $14.01 \pm 0.07$ \\
\hline Q2 & $13.99 \pm 0.06$ & Q2 & $13.95 \pm 0.06$ \\
\hline Q3 & $14.07 \pm 0.06$ & $\widehat{\mathrm{Q}} 3$ & $14.01 \pm 0.07$ \\
\hline $\mathrm{Q} 4$ & $13.98 \pm 0.07$ & $\mathrm{Q} 4$ & $14.03 \pm 0.07$ \\
\hline$p$ for trend & 0.621 & $p$ for trend & 0.646 \\
\hline
\end{tabular}

Data are presented as mean \pm SE.

Subgroup analyses showed that for subjects with IOP $\leq 21 \mathrm{mmHg}(n=767,98.9 \%$ of total glaucoma patients), only lower niacin intake was associated with a higher odds ratio for glaucoma $(p=0.022 ;$ Table 6). 
Table 6. Odds ratios of nutritional factors for glaucoma in subjects with intraocular pressure $\leq 21 \mathrm{mmHg}$.

\begin{tabular}{|c|c|c|c|c|c|}
\hline Nutrient & OR $(95 \%$ CI $)$ & $p$ for Trend & Nutrient & OR $(95 \%$ CI $)$ & $p$ for Trend \\
\hline Crude fiber & & \multirow{5}{*}{0.311} & Vitamin A & & \multirow{5}{*}{0.161} \\
\hline Q1 & Reference & & Q1 & Reference & \\
\hline$\hat{\mathrm{Q} 2}$ & $0.99(0.77-1.21)$ & & $\hat{\mathrm{Q}} 2$ & $1.01(0.80-1.28)$ & \\
\hline Q3 & $0.92(0.71-1.21)$ & & Q3 & $0.94(0.72-1.24)$ & \\
\hline$\widehat{Q} 4$ & $0.87(0.66-1.17)$ & & Q4 & $0.82(0.62-1.09)$ & \\
\hline Ash & & \multirow{5}{*}{0.438} & $\beta$-Carotene & & \multirow{5}{*}{0.251} \\
\hline Q1 & Reference & & Q1 & Reference & \\
\hline Q2 & $0.82(0.62-1.08)$ & & Q2 & $0.98(0.77-1.24)$ & \\
\hline Q3 & $0.98(0.73-1.30)$ & & Q3 & $0.89(0.68-1.18)$ & \\
\hline $\mathrm{Q} 4$ & $0.80(0.56-1.16)$ & & $\mathrm{Q} 4$ & $0.86(0.66-1.13)$ & \\
\hline Calcium & & \multirow{5}{*}{0.635} & Retinol & & \multirow{5}{*}{0.210} \\
\hline Q1 & Reference & & Q1 & Reference & \\
\hline Q2 & $0.91(0.69-1.19)$ & & Q2 & 1.17 (0.92-1.48) & \\
\hline$\widehat{Q} 3$ & $0.74(0.56-0.99)$ & & $\widehat{\mathrm{Q} 3}$ & $0.99(0.76-1.28)$ & \\
\hline Q4 & $0.99(0.73-1.35)$ & & Q4 & $0.86(0.64-1.15)$ & \\
\hline Phosphorus & & \multirow{5}{*}{0.440} & Thiamin & & \multirow{5}{*}{0.428} \\
\hline $\mathrm{Q} 1$ & Reference & & Q1 & Reference & \\
\hline Q2 & $0.88(0.67-1.15)$ & & Q2 & $0.98(0.75-1.30)$ & \\
\hline Q3 & $0.85(0.62-1.17)$ & & Q3 & $0.85(0.62-1.17)$ & \\
\hline$\widehat{Q} 4$ & $0.86(0.55-1.34)$ & & $\widehat{\mathrm{Q}} 4$ & $0.90(0.61-1.33)$ & \\
\hline Iron & & \multirow{5}{*}{0.091} & Riboflavin & & \multirow{5}{*}{0.154} \\
\hline Q1 & Reference & & Q1 & Reference & \\
\hline$\widehat{\mathrm{Q}} 2$ & 0.77 (0.59-0.99) & & $\widehat{\mathrm{Q}} 2$ & $0.91(0.70-1.18)$ & \\
\hline Q3 & $0.82(0.61-1.12)$ & & Q3 & $0.77(0.57-1.04)$ & \\
\hline Q4 & $0.73(0.53-1.01)$ & & $\widehat{Q} 4$ & $0.82(0.56-1.18)$ & \\
\hline Sodium & & \multirow{5}{*}{0.364} & Niacin & & \multirow{5}{*}{0.022} \\
\hline Q1 & Reference & & Q1 & Reference & \\
\hline$\hat{\mathrm{Q} 2}$ & $1.01(0.77-1.31)$ & & Q2 & $0.82(0.63-1.09)$ & \\
\hline Q3 & $1.25(0.96-1.62)$ & & Q3 & $0.71(0.51-0.99)$ & \\
\hline$\widehat{Q} 4$ & $1.06(0.78-1.46)$ & & $\widehat{Q} 4$ & $0.63(0.41-0.96)$ & \\
\hline Potassium & & \multirow{5}{*}{0.128} & Vitamin C & & \multirow{5}{*}{0.455} \\
\hline Q1 & Reference & & Q1 & Reference & \\
\hline Q2 & $0.90(0.69-1.19)$ & & Q2 & $1.10(0.85-1.41)$ & \\
\hline$\hat{\mathrm{Q} 3}$ & $0.86(0.62-1.18)$ & & $\widehat{Q} 3$ & $0.98(0.74-1.29)$ & \\
\hline $\mathrm{Q} 4$ & $0.74(0.50-1.08)$ & & $\mathrm{Q} 4$ & $0.91(0.68-1.23)$ & \\
\hline
\end{tabular}

Data are presented OR $(95 \%$ CI). Statistics were carried out using Logistic regression. Odds ratios are adjusted for age, gender, income, education, occupation, smoking, drink, physical activity, diabetes, hypertension, intraocular pressure, total energy.

After the exclusion of individuals taking supplements, higher intake of riboflavin $(p=0.009)$ and niacin $(p=0.035)$ were related to a lower risk of glaucoma (Table 7). Among subjects without glaucoma, 4100 subjects $(25.6 \%)$ were taking supplements. Among individuals with glaucoma, 193 individuals (24.9\%) were taking supplements. 
Table 7. Odds ratios of nutritional factors for glaucoma after exclusion of participants taking supplements.

\begin{tabular}{|c|c|c|c|c|c|}
\hline Nutrient & OR $(95 \%$ CI $)$ & $p$ for Trend & Nutrient & OR $(95 \%$ CI $)$ & $p$ for Trend \\
\hline Crude fiber & & \multirow{5}{*}{0.410} & Vitamin A & & \multirow{5}{*}{0.481} \\
\hline Q1 & Reference & & Q1 & Reference & \\
\hline$\widehat{\mathrm{Q} 2}$ & $0.96(0.73-1.28)$ & & Q2 & $0.92(0.70-1.21)$ & \\
\hline Q3 & $0.88(0.64-1.20)$ & & Q3 & $0.95(0.69-1.30)$ & \\
\hline$\widehat{Q} 4$ & $0.90(0.65-1.24)$ & & Q4 & $0.87(0.63-1.21)$ & \\
\hline Ash & & \multirow{5}{*}{0.377} & $\beta$-Carotene & & \multirow{5}{*}{0.505} \\
\hline Q1 & Reference & & Q1 & Reference & \\
\hline Q2 & $0.84(0.61-1.16)$ & & Q2 & $0.94(0.71-1.25)$ & \\
\hline Q3 & $0.91(0.65-1.27)$ & & Q3 & $0.89(0.65-1.22)$ & \\
\hline $\mathrm{Q} 4$ & $0.80(0.53-1.21)$ & & $\mathrm{Q} 4$ & $0.91(0.67-1.24)$ & \\
\hline Calcium & & \multirow{5}{*}{0.618} & Retinol & & \multirow{5}{*}{0.125} \\
\hline Q1 & Reference & & Q1 & Reference & \\
\hline$\widehat{\mathrm{Q}} 2$ & $0.91(0.65-1.26)$ & & Q2 & $1.12(0.85-1.48)$ & \\
\hline$\widehat{\mathrm{Q} 3}$ & $0.78(0.56-1.08)$ & & $\widehat{\mathrm{Q} 3}$ & $0.91(0.68-1.21)$ & \\
\hline $\mathrm{Q} 4$ & $0.96(0.67-1.37)$ & & $\mathrm{Q} 4$ & $0.82(0.60-1.13)$ & \\
\hline Phosphorus & & \multirow{5}{*}{0.419} & Thiamin & & \multirow{5}{*}{0.267} \\
\hline $\mathrm{Q} 1$ & Reference & & Q1 & Reference & \\
\hline Q2 & $0.83(0.60-1.15)$ & & Q2 & $0.92(0.67-1.25)$ & \\
\hline Q3 & $0.82(0.56-1.20)$ & & Q3 & $0.89(0.62-1.29)$ & \\
\hline Q4 & $0.82(0.47-1.40)$ & & $\widehat{\mathrm{Q}} 4$ & $0.76(0.48-1.20)$ & \\
\hline Iron & & \multirow{5}{*}{0.0502} & Riboflavin & & \multirow{5}{*}{0.009} \\
\hline Q1 & Reference & & Q1 & Reference & \\
\hline$\widehat{\mathrm{Q}} 2$ & $0.70(0.52-0.94)$ & & $\widehat{\mathrm{Q}} 2$ & $0.81(0.60-1.08)$ & \\
\hline Q3 & $0.88(0.63-1.23)$ & & Q3 & $0.63(0.44-0.89)$ & \\
\hline$\widehat{\mathrm{Q}} 4$ & $0.64(0.44-0.91)$ & & $\mathrm{Q} 4$ & $0.60(0.39-0.93)$ & \\
\hline Sodium & & \multirow{5}{*}{0.424} & Niacin & & \multirow{5}{*}{0.035} \\
\hline Q1 & Reference & & Q1 & Reference & \\
\hline Q2 & $1.00(0.74-1.35)$ & & Q2 & $0.73(0.52-1.02)$ & \\
\hline Q3 & $1.26(0.94-1.69)$ & & Q3 & $0.75(0.51-1.12)$ & \\
\hline$\widehat{\mathrm{Q}} 4$ & $1.05(0.73-1.50)$ & & $\widehat{Q} 4$ & $0.54(0.33-0.90)$ & \\
\hline Potassium & & \multirow{5}{*}{0.100} & Vitamin C & & \multirow{5}{*}{0.726} \\
\hline Q1 & Reference & & Q1 & Reference & \\
\hline Q2 & $0.70(0.51-0.96)$ & & Q2 & $1.14(0.86-1.53)$ & \\
\hline$\widehat{Q} 3$ & $0.75(0.53-1.07)$ & & $\widehat{Q} 3$ & $1.01(0.75-1.36)$ & \\
\hline $\mathrm{Q} 4$ & $0.68(0.44-1.04)$ & & $\mathrm{Q} 4$ & $1.11(0.79-1.55)$ & \\
\hline
\end{tabular}

Data are presented OR $(95 \% \mathrm{CI})$. Statistics were carried out using Logistic regression, adjusted for age, gender, income, education, occupation, smoking, drink, Physical activity, diabetes, hypertension, Intraocular pressure, total energy.

\section{Discussion}

We demonstrated that dietary intake of niacin was associated with glaucoma, independent of IOP. Mean IOP was similar by quartiles of all examined nutrients. Individuals with NTG showed lower intake of niacin among nutrients. After the exclusion of individuals taking supplements, higher intake of riboflavin and niacin were related to a lower risk of glaucoma. Overall, lower intake of niacin remained significantly associated with glaucoma also in the subgroup analysis.

Previously, some reports found that low intake of vitamins was associated with augmented risk for glaucoma [11,13]. The Rotterdam study, a prospective population-based study, reported that a low intake of retinol equivalents and vitamin B1 appeared to increase the risk for POAG [11]. Wang et al. suggested that supplementary ingestion of vitamin $C$ was associated with reduced glaucoma risk [13]. The Nurses' Health Study and Health Professionals Follow-up Study did not detect a correlation between nutrients with anti-oxidant properties and open-angle glaucoma [14]. However, the diagnosis 
of glaucoma was based on self-report, even though a strength of that study was that it was a large prospective study [14]. This could result in a selection bias because over half of individuals with glaucoma are not aware of their condition [21,22].

IOP is the most critical risk factor for glaucoma, even though glaucoma can develop in cases with a normal range of IOP. We analyzed IOP distribution by quartiles of dietary nutrients and found no significant differences in mean IOP according to dietary nutrients. The relationship between nutrients and glaucoma was analyzed adjusting for IOP to remove the influence of dietary nutrient intake on IOP. There was an association between dietary intake of niacin with glaucoma, independent of IOP.

With regard to NTG, only one Japanese study observed that lower serum vitamin C levels were correlated with increased risk of NTG [23]. The authors speculated that glutamate-stimulated release of vitamin $C$ might decrease oxidative stress induced by glutamate excitotoxicity and reduce the degeneration of retinal ganglion cells [23]. The difference between that study and this study is that only vitamin A, B9, C, E and uric acid were investigated using serum samples, and niacin was not included in that study [23].

In this study, only dietary niacin intake was lower in subjects with NTG. In addition, niacin remained as the factor associated with glaucoma after the exclusion of subjects taking supplements. Recently, Williams et al. found that oral administration of vitamin B3 protected retinal ganglion cells in aged mouse with chronic ocular hypertension, modulating mitochondrial vulnerability [24]. In a stroke animal model, niacin (nicotinic acid) treatment promoted synaptic plasticity and axon growth [25]. That study suggested that brain-derived neurotrophic factor (BDNF)/tropomyosin receptor kinase $B(\operatorname{TrKB})$ pathways seemed to be involved in niacin-induced neuroprotective effects after a stroke [25]. Disrupted axonal transport of neurotrophic factors is one of main mechanisms of glaucoma [3]. Therefore, upregulated BDNF by niacin treatment might decrease the risk of glaucoma. Kaplon et al. reported that niacin intake was positively correlated with better vascular endothelial function associated with decreased vascular oxidative stress [26]. The vascular hypothesis for glaucoma pathogenesis states insufficient or unstable blood supply is a key contributing factor for glaucoma development or progression [27]. A collaborative normal tension glaucoma study showed vascular factors, such as disc hemorrhage and migraine, were risk factors for the progression of glaucoma [28]. Compromised vascular endothelial cell function has been reported in subjects with NTG $[29,30]$. Therefore, it appears that improved vascular endothelial cell function induced by niacin could lower the risk for open-angle glaucoma. To the best of our knowledge, this is the first study to reveal the relationship between niacin and glaucoma in human. However, the study was simply descriptive and inconclusive on disease mechanisms. Measurement of serum or intravitreal level of niacin in glaucoma patients would be helpful to understand the mechanism of how low niacin influences glaucoma.

The strength of this population-based study is that study participants were representative of subjects with glaucoma in the general population. We present the first study analyzing the relationship between dietary nutrients and glaucoma (especially NTG) in an Asian country. One limitation of this study is that we could not determine cause and effect for the relationship between dietary nutrients and glaucoma. Prospective randomized controlled trial or epidemiological cohort studies are needed to ensure the usefulness of niacin in glaucoma. Another limitation is that the nutritional survey was conducted just once, although all subjects were educated to continue their ordinary diets before the dietary evaluation. There might be a confounding effect of seasonal foods because all participants did not take part in the nutritional survey at the same time of year. The proportion of participants taking dietary reference intakes for Koreans (KDRIs) was relatively lower for calcium $(67.0 \pm 0.8 \%)$ and potassium $(61.9 \pm 0.6 \%)$, according to data from the Korea National Health and Nutrition Examination Survey (2012). Therefore, the association of glaucoma with the intake of these nutrients might be underestimated. Nutrient intake was calculated on the basis of the nutrient concentrations in foods using the Korean Food Composition Table. Potential errors can exist in the tables that describe foods. The errors may result in inaccurate measurement of nutrient intake at the individual level, though probably less so at the group level. The Korean Food Composition Tables cover nearly 3000 food items 
in 19 groups, being almost complete for nutritional evaluation of daily foods. Kim et al. reported the accuracy of conventional food composition table-based estimation of intakes of protein, lipid and carbohydrate, in comparison with chemical analysis [31]. Their result supports that the Korean Food Composition Tables are sufficiently accurate, even though that study did not evaluate the accuracy of niacin. Analysis from dietary nutrients has limitations because the bioavailability of nutrients may vary in each individual. Further, serum analysis of nutrients may be needed to investigate the direct association of glaucoma and nutrients.

\section{Conclusions}

In conclusion, this population-based study found that lower nutrient intake of niacin was associated with glaucoma. Lower niacin intake was associated with NTG. In addition, niacin remained as the factor related to glaucoma after the exclusion of subjects taking supplements. Glaucoma is a progressive neurodegenerative disease and can lead to vision loss, despite a substantial decrease in IOP. The prevention of glaucoma is important, because neurodegeneration of retinal ganglion cells is irreversible. Dietary nutrition is a modifiable factor; the discovery that nutrition can help decrease glaucoma development or progression may help individuals with glaucoma even though this needs confirmation from longitudinal, prospective studies.

Acknowledgments: This research was supported by the Basic Science Research Program through the National Research Foundation of Korea (NRF) funded by the Ministry of Education (K.I.J., 2017R1D1A1B03035355). Statistical consultation was supported by a grant from the Korean Health Technology R\&D Project through the Korean Health Industry Development Institute (KHIDI), funded by the Ministry of Health \& Welfare, Republic of Korea (grant number: HI14C1062). The authors thank Ki Ho Park, Department of Ophthalmology, Seoul National University College of Medicine and Chan Yun Kim, Department of Ophthalmology, Institute of Vision Research, Yonsei University College of Medicine for reviewing this article. We report these results on behalf of the Epidemiologic Survey Committee of the Korean Ophthalmological Society.

Author Contributions: Designed the study: K.I.J., C.K.P.; Carried out the study: K.I.J.; Analyzed the data: K.I.J., Y.C.K., C.K.P.; Wrote the article: K.I.J.

Conflicts of Interest: The authors declare that no competing interests exist with the funder. The funders had no role in study design, data collection and analysis, decision to publish, or preparation of the manuscript.

\section{References}

1. Quigley, H.A.; Broman, A.T. The number of people with glaucoma worldwide in 2010 and 2020. Br. J. Ophthalmol. 2006, 90, 262-267. [CrossRef] [PubMed]

2. Resnikoff, S.; Pascolini, D.; Etya'Ale, D.; Kocur, I.; Pararajasegaram, R.; Pokharel, G.P.; Mariotti, S.P. Global data on visual impairment in the year 2002. Bull. World Health Org. 2004, 82, 844-851. [PubMed]

3. Weinreb, R.N.; Aung, T.; Medeiros, F.A. The pathophysiology and treatment of glaucoma: A review. JAMA 2014, 311, 1901-1911. [CrossRef] [PubMed]

4. Tielsch, J.M.; Katz, J.; Sommer, A.; Quigley, H.A.; Javitt, J.C. Family history and risk of primary open angle glaucoma: The Baltimore eye survey. Arch. Ophthalmol. 1994, 112, 69-73. [CrossRef] [PubMed]

5. Abu-Amero, K.; Kondkar, A.A.; Chalam, K.V. An updated review on the genetics of primary open angle glaucoma. Int. J. Mol. Sci. 2015, 16, 28862-28911. [CrossRef] [PubMed]

6. Mudumbai, R.C. Clinical update on normal tension glaucoma. Semin. Ophthalmol. 2013. [CrossRef] [PubMed]

7. Iwase, A.; Suzuki, Y.; Araie, M.; Yamamoto, T.; Abe, H.; Shirato, S.; Kuwayama, Y.; Mishima, H.K.; Shimizu, H.; Tomita, G.; et al. The prevalence of primary open-angle glaucoma in Japanese: The Tajimi Study. Ophthalmology 2004, 111, 1641-1648. [CrossRef]

8. Pekmezci, M.; Vo, B.; Lim, A.K.; Hirabayashi, D.R.; Tanaka, G.H.; Weinreb, R.N.; Lin, S.C. The characteristics of glaucoma in Japanese Americans. Arch. Ophthalmol. 2009, 127, 167-171. [CrossRef] [PubMed]

9. Coleman, A.L.; Stone, K.L.; Kodjebacheva, G.; Yu, F.; Pedula, K.L.; Ensrud, K.E.; Cauley, J.A.; Hochberg, M.C.; Topouzis, F.; Badala, F.; et al. Glaucoma risk and the consumption of fruits and vegetables among older women in the study of osteoporotic fractures. Am. J. Ophthalmol. 2008, 145, 1081-1089. [CrossRef] [PubMed] 
10. Kang, J.H.; Pasquale, L.R.; Willett, W.C.; Rosner, B.A.; Egan, K.M.; Faberowski, N.; Hankinson, S.E. Dietary fat consumption and primary open-angle glaucoma. Am. J. Clin. Nutr. 2004, 79, 755-764. [CrossRef] [PubMed]

11. Ramdas, W.D.; Wolfs, R.C.; Kiefte-de Jong, J.C.; Hofman, A.; de Jong, P.T.; Vingerling, J.R.; Jansonius, N.M. Nutrient intake and risk of open-angle glaucoma: The Rotterdam Study. Eur. J. Epidemiol. 2012, 27, 385-393. [CrossRef] [PubMed]

12. Renard, J.P.; Rouland, J.F.; Bron, A.; Sellem, E.; Nordmann, J.P.; Baudouin, C.; Denis, P.; Villain, M.; Chaine, G.; Colin, J.; et al. Nutritional, lifestyle and environmental factors in ocular hypertension and primary open-angle glaucoma: An exploratory case-control study. Acta Ophthalmol. 2013, 91, 505-513. [CrossRef] [PubMed]

13. Wang, S.Y.; Singh, K.; Lin, S.C. Glaucoma and vitamins A, C, and E supplement intake and serum levels in a population-based sample of the United States. Eye. Lond. 2013, 27, 487-494. [CrossRef] [PubMed]

14. Kang, J.H. Antioxidant intake and primary open-angle glaucoma: A prospective study. Am. J. Epidemiol. 2003, 158, 337-346. [CrossRef] [PubMed]

15. Li, S.; Li, D.; Shao, M.; Cao, W.; Sun, X. Lack of association between serum vitamin B6, vitamin B12, and vitamin D levels with different types of glaucoma: A systematic review and meta-analysis. Nutrients 2017, 9, 636. [CrossRef] [PubMed]

16. Engin, K.N. Alpha-tocopherol: Looking beyond an antioxidant. Mol. Vis. 2009, 15, 855-860. [PubMed]

17. Ritch, R. Neuroprotection: Is it already applicable to glaucoma therapy? Curr. Opin. Ophthalmol. 2000, 11, 78-84. [CrossRef] [PubMed]

18. Foster, P.J.; Buhrmann, R.; Quigley, H.A.; Johnson, G.J. The definition and classification of glaucoma in prevalence surveys. Br. J. Ophthalmol. 2002, 86, 238-242. [CrossRef] [PubMed]

19. Kim, D.W.; Kim, Y.K.; Jeoung, J.W.; Kim, D.M.; Park, K.H. Prevalence of optic disc hemorrhage in Korea: The Korea National Health and Nutrition Examination Survey. Investig. Ophthalmol. Vis. Sci. 2015, 56, 3666-3672. [CrossRef] [PubMed]

20. Rural Development Administration. Food Composition Table, 7th ed.; Rural Development Administration: Suwon, Republic of Korea, 2006.

21. Coleman, A.L. Glaucoma. Lancet 1999, 354, 1803-1810. [CrossRef]

22. Jung, K.I.; Park, C.K. Mental health status and quality of life in undiagnosed glaucoma patients: A nationwide population-based study. Medicine (Baltimore) 2016, 95, e3523. [CrossRef] [PubMed]

23. Yuki, K.; Murat, D.; Kimura, I.; Ohtake, Y.; Tsubota, K. Reduced-serum vitamin C and increased uric acid levels in normal-tension glaucoma. Graefes Arch. Clin. Exp. Ophthalmol. 2010, 248, 243-248. [CrossRef] [PubMed]

24. Williams, P.A.; Harder, J.M.; Foxworth, N.E.; Cochran, K.E.; Philip, V.M.; Porciatti, V.; Smithies, O.; John, S.W. Vitamin B3 modulates mitochondrial vulnerability and prevents glaucoma in aged mice. Science 2017, 355, 756-760. [CrossRef] [PubMed]

25. Cui, X.; Chopp, M.; Zacharek, A.; Roberts, C.; Buller, B.; Ion, M.; Chen, J. Niacin treatment of stroke increases synaptic plasticity and axon growth in rats. Stroke 2010, 41, 2044-2049. [CrossRef] [PubMed]

26. Kaplon, R.E.; Gano, L.B.; Seals, D.R. Vascular endothelial function and oxidative stress are related to dietary niacin intake among healthy middle-aged and older adults. J. Appl. Physiol. 2014, 116, 156-163. [CrossRef] [PubMed]

27. Flammer, J. The vascular concept of glaucoma. Surv. Ophthalmol. 1994, 38, S3-S6. [CrossRef]

28. Drance, S.; Anderson, D.R.; Schulzer, M. Risk factors for progression of visual field abnormalities in normal-tension glaucoma. Am. J. Ophthalmol. 2001, 131, 699-708. [CrossRef]

29. Henry, E.; Newby, D.E.; Webb, D.J.; O’Brien, C. Peripheral endothelial dysfunction in normal pressure glaucoma. Invest. Ophthalmol. Vis. Sci. 1999, 40, 1710-1714. [PubMed] 
30. Buckley, C.; Hadoke, P.W.; Henry, E.; O’Brien, C. Systemic vascular endothelial cell dysfunction in normal pressure glaucoma. Br. J. Ophthalmol. 2002, 86, 227-232. [CrossRef] [PubMed]

31. Kim, E.S.; Ko, Y.S.; Kim, J.; Matsuda-Inoguchi, N.; Nakatsuka, H.; Watanabe, T.; Shimbo, S.; Ikeda, M. Food composition table-based estimation of energy and major nutrient intake in comparison with chemical analysis: A validation study in Korea. Tohoku J. Exp. Med. 2003, 200, 7-15. [CrossRef] [PubMed] 